Annales Geophysicae (2001) 19: 147-157 C European Geophysical Society 2001

\title{
LHR effects in nonducted whistler propagation - new observations and numerical modelling
}

\author{
F. Jiříček ${ }^{1}$, D. R. Shklyar ${ }^{2}$, and P. Tř́íska ${ }^{1}$ \\ ${ }^{1}$ Institute of Atmospheric Physics, Acad. Sci. Czech Republic, Boční II, 14131 Prague 4, Czech Republic \\ ${ }^{2}$ IZMIRAN, Troitsk, Moscow Region, 142190, Russia
}

Received: 10 May 2000 - Revised: 27 November 2000 - Accepted: 8 December 2000

\begin{abstract}
VLF-ELF broadband measurements onboard the MAGION 4 and 5 satellites at heights above 1 Re in plasmasphere provide new data on various known phenomena related to ducted and nonducted whistler wave propagation. Two examples are discussed: magnetospherically reflected (MR) whistlers and lower hybrid resonance (LHR) noise band. We present examples of rather complicated MR whistler spectrograms not reported previously and argue the conditions for their generation. Analytical consideration, together with numerical modelling, yield understanding of the main features of those spectrograms. LHR noise band, as well as MR whistlers, is a phenomenon whose source is the energy propagating in the nonducted way. At the plasmaspheric heights, where hydrogen $\left(\mathrm{H}^{+}\right)$is the prevailing ion, and electron plasma frequency is much larger than gyrofrequency, the LHR frequency is close to its maximum value in a given magnetic field. This frequency is well followed by the observed noise bands. The lower cutoff frequency of this band is somewhat below that maximum value. The reason for this, as well as the possibility of using the LHR noise bands for locating the plasma through position, are discussed.
\end{abstract}

Key words. Magnetospheric physics (plasmasphere; wave propagation)

\section{Introduction}

Broadband (VLF-ELF, $f<22.5 \mathrm{kHz}$ ) electromagnetic wave measurements onboard the MAGION 4 and 5 satellites include electric and magnetic field components registered by a double-probe dipole $1.7 \mathrm{~m}$ long and by magnetic searchcoils, respectively. These measurements contain most of the known phenomena related to ducted and nonducted propagation of whistler mode waves:

Correspondence to: F. Jiříček (fji@ ufa.cas.cz)
- plasmaspheric hiss, chorus, discrete plasmaspheric emissions;

- ducted and nonducted whistlers;

- lower hybrid resonance (LHR) noise band and LHR whistlers;

- magnetospherically reflected (MR) whistlers.

The literature devoted to all these types of emissions is extensive. We refer to the review by Sazhin (1982) where the references to many other papers can be found. For discussion in the present study, we have chosen two phenomena closely connected with LHR frequency of the medium: MR whistlers first observed by Smith and Angerami (1968) aboard OGO 1 and LHR noise (Barrington et al., 1963; Brice and Smith, 1965). These two phenomena are often found in the data from MAGION 4 and 5.

The importance of LHR effects for understanding whistler wave propagation in the magnetosphere has first been established by Kimura (1966) who has shown that, with the account of LHR effects, the whistler mode wave can be reflected from the magnetospheric (or upper ionospheric) region where the LHR frequency of the medium exceeds the wave frequency. As a consequence, the wave can be trapped inside the region with a minimum in LHR frequency profile, in particular, on the closed field lines in the magnetosphere with the LHR minimum at the equator, or in some special regions in the upper ionosphere where a minimum of LHR frequency profile may be formed due to specific behaviour of the ion effective mass. A mechanism of quasi-electrostatic wave trapping in LHR waveguide in the upper ionosphere has been investigated by Jiř́iček and Shklyar (1999).

Kimura's idea of LHR reflection of whistler mode waves propagating in the magnetosphere is the key point in understanding of MR whistler trace formation. This understanding has been achieved in basic studies of MR whistlers by Smith and Angerami (1968), Edgar (1976), and Thorne and Horne (1994). The ideas of these authors have been used by Shklyar and Jiříček (2000) for numerical simulation of various types of MR whistler spectrograms observed by MAGION 4 and 5. 
Since MR whistlers and LHR noise bands were discovered, there were suggestions that LHR waves could sometimes be excited by whistlers in the magnetosphere (Barrington et al., 1963; Brice and Smith, 1964; Helliwell, 1965; see also the review by Jiříček and Tř́íska, 1976; the paper by Bell et al., 1991, and references to previous works therein). In particular, the coupling of whistlers and LHR waves was found in VLF transmitter signal broadening attributed to LHR wave excitation by a transmitter signal (Titova et al., 1984; Bell and Ngo, 1988). An important point in the wave coupling is the recent experimental observation by Bell et al. (1991) that LHR waves are effectively excited by whistlers in magnetospheric regions where small scale plasma density irregularities exist. Thus, the wave coupling responsible for signal spectral broadening involves VLF wave scattering on plasma density irregularities. These irregularities could be connected with other waves, making this coupling a nonlinear effect. Here we discuss another aspect of the relationship between MR whistlers and LHR noise which is present in the absence of linear or nonlinear wave conversion.

\section{Observations of nonducted whistler waves from MAGION 4 and 5}

\subsection{MR whistlers}

A type of MR whistler spectrogram, first observed onboard OGO 1 (Smith and Angerami, 1968), has also been registered by MAGION 4 and 5 when the satellites crossed the equatorial region of magnetosphere at $L$-shells below 3. Examples of such spectrograms from MAGION 4 and 5 data are shown in Fig. 1. However, the analysis of large amounts of data on MR whistlers from MAGION 4 and 5 shows that this type of spectrogram is not the most frequently found in the data. More often, other types of MR whistler spectograms are observed, few examples of which are shown in Fig. 2. Furthermore, Fig. 3 provides examples of rather complicated spectrograms observed by the MAGION 4 satellite.

During quiet magnetospheric conditions, MR whistlers are typically registered in the equatorial region of magnetosphere at $L$-shells from about 2.1 to 3.1. The region where MR whistlers were found by MAGION 4 is shown in Fig. 4. Observations from MAGION 5 confirm this result, and also show that MR whistlers are most frequently found near $L$ shell $\simeq 2.5$. Although MR whistlers are related to nonducted propagation and, thus, their source region cannot be easily determined from the observation point and the spectrogram pattern, in some particular cases, ducted whistlers were observed simultaneously with MR whistlers, which gave an idea about the exit region of the waves. It should be stressed that the first trace of MR whistlers which is formed by halfhop waves may be very close to a ducted whistler trace, thus, one should be very careful in distinguishing between them.

Analysis of complex spectrograms found in experimental data shows that their peculiar pattern is connected with specific illuminating region and depends essentially on the ob-
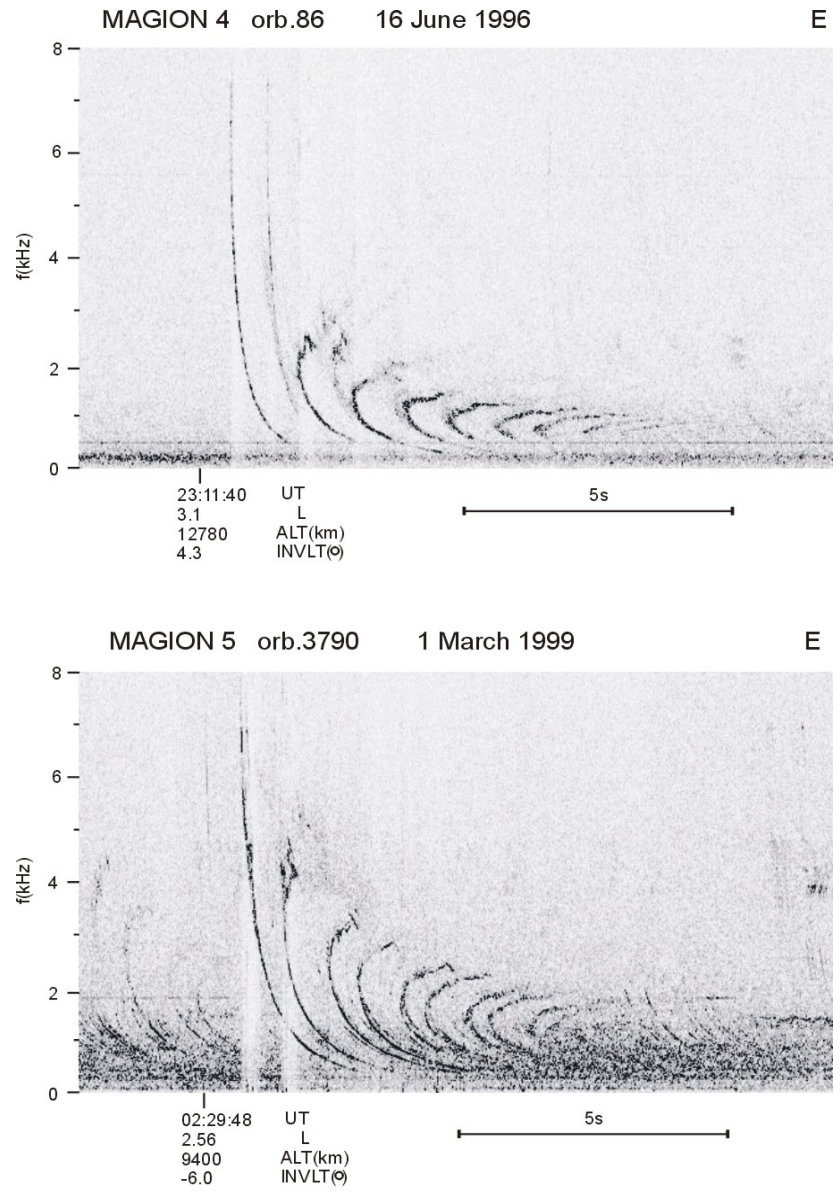

Fig. 1. Examples of MAGION 4 and 5 MR whistler registrations of similar type as observed onboard OGO 1. Positive and negative INVLT correspond to the Northern and the Southern hemispheres, respectively.

servation point and geomagnetic activity. Large amounts of experimental data available from MAGION 4 and 5 measurements provide evidences that the existence of extended plasmasphere is a necessary condition for the formation of MR whistlers. This follows from the observation that the probability of MR whistler occurrence decreases with increasing geomagnetic activity when the plasmapause approaches lower $L$-shells. In Sect. 4 , we will model this effect by taking into account the plasmapause in simulations. We should mention that the role of sharp cross- $L$ density drop-off along a particular field line in formation of upper-frequency cutoff on MR whistler spectrogram has been considered by Edgar (1976).

\subsection{LHR noise}

The noise band at the lower hybrid resonance, known as LHR noise (or LHR hiss), has been detected in the VLF recordings in the plasmaspheric regions of the magnetosphere, beginning from the observations of the Alouette satellite. The few references listed below by no means represent all the signif- 

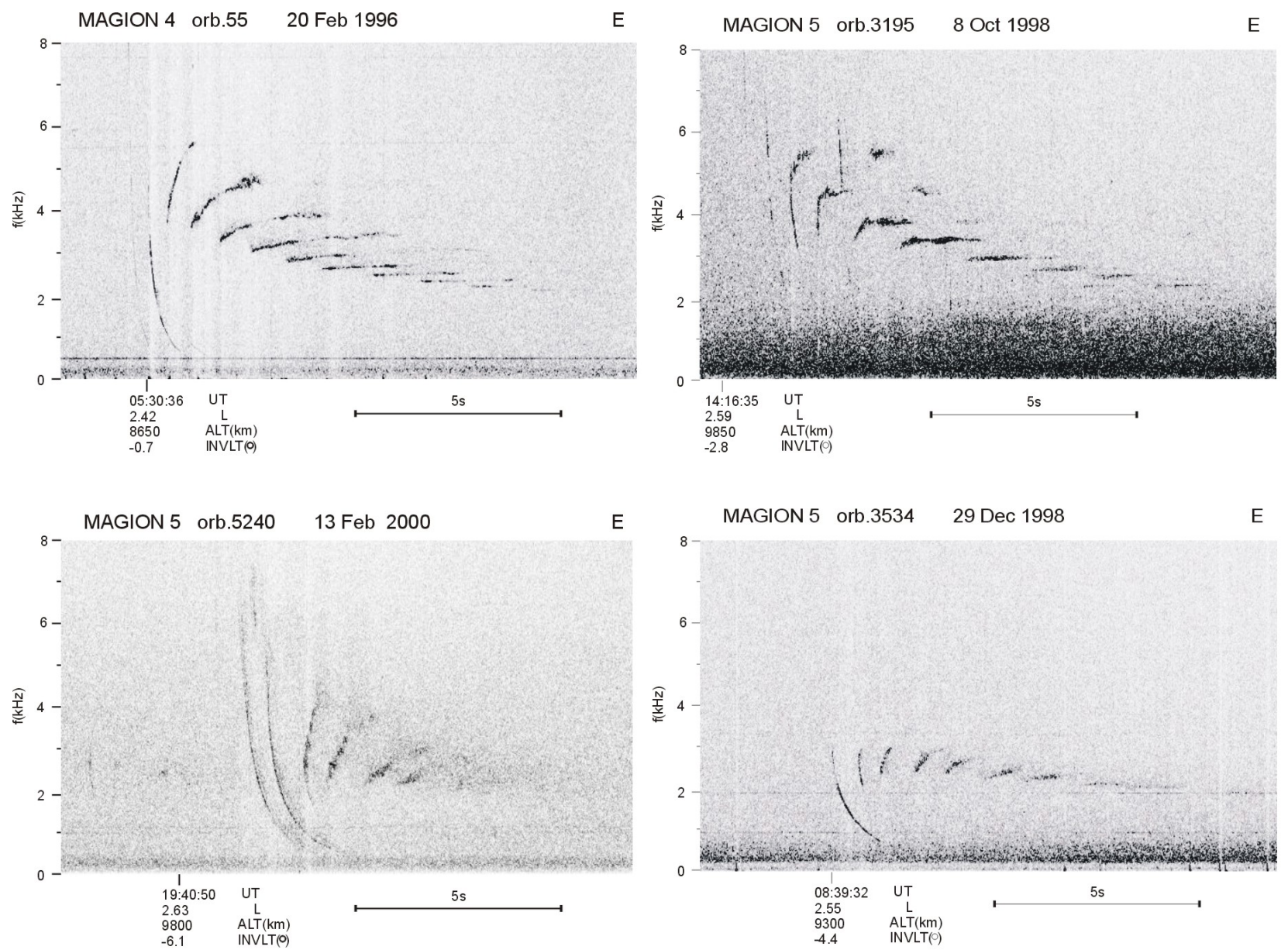

Fig. 2. Types of MR whistler spectrograms most frequently received at MAGION 4 and 5.

icant works on this subject, but could serve as milestones in the investigations of this phenomena (Barrington and Belrose, 1963; Brice and Smith, 1965; Laaspere et al., 1971; Burtis, 1973). Based on the papers listed above, important features of LHR noise can be summarized as follows:

- sharp lower frequency cutoff that in general varies with varying satellite position;

- connection with nonducted whistlers and magnetospheric reflections;

- predominant observation with electric antennas;

- triggering of LHR noise by magnetospherically reflected whistlers.

Figure 5 shows examples of LHR noise along MAGION 5 orbits. The lower cutoff frequency of the band follows the LHR frequency $f_{\text {LHR }}$. The curve of $f_{\text {LHRmax }}$ (see Sect. 5) is shown by a dashed line. Close to the end of this record, the satellite approaches the plasmapause. The effect of the plasmapause is a little more distinctly expressed in the lower panel of Fig. 5. The decrease of $f_{\mathrm{LHR}}$ at about 04:18 UT together with occurrence of chorus above $4 \mathrm{kHz}$ are typical signatures of plasmapause crossing. In the case shown in the bottom panel of Fig. 5, the satellite leaves the plasmasphere after the sudden commencement of magnetic disturbance (SSC). Arrow 1 shows the location of the plasmapause before SSC, determined by $K_{p}$ index, according to the Carpenter and Anderson (1992) model. Arrow 2 shows the location of the plasmapause at the time of observation corresponding to an increasing magnetic disturbance. This illustrates how the location of the region where LHR noise is observed depends on magnetic disturbances and the related location of the plasmapause; the observation of LHR noise is usually ceased near the plasmapause.

A short interval from the record of electric component displayed in the upper panel of Fig. 5 is presented in Fig. 6 as VLF spectrogram detected with electric and magnetic antennas (upper and lower panels, respectively). While usual whistlers are seen in both panels, LHR noise is registered only by the electric antenna. This agrees with the well known fact that LHR noise represents a type of quasi-electrostatic wave. Figure 7 displays 28 seconds from the electric field spectrum, shown in the top panel of Fig. 5, and clearly demonstrates a close connection of the observed LHR noise with the whistler mode wave energy propagating in nonducted mode, namely, with diffusive whistler traces exhibit- 

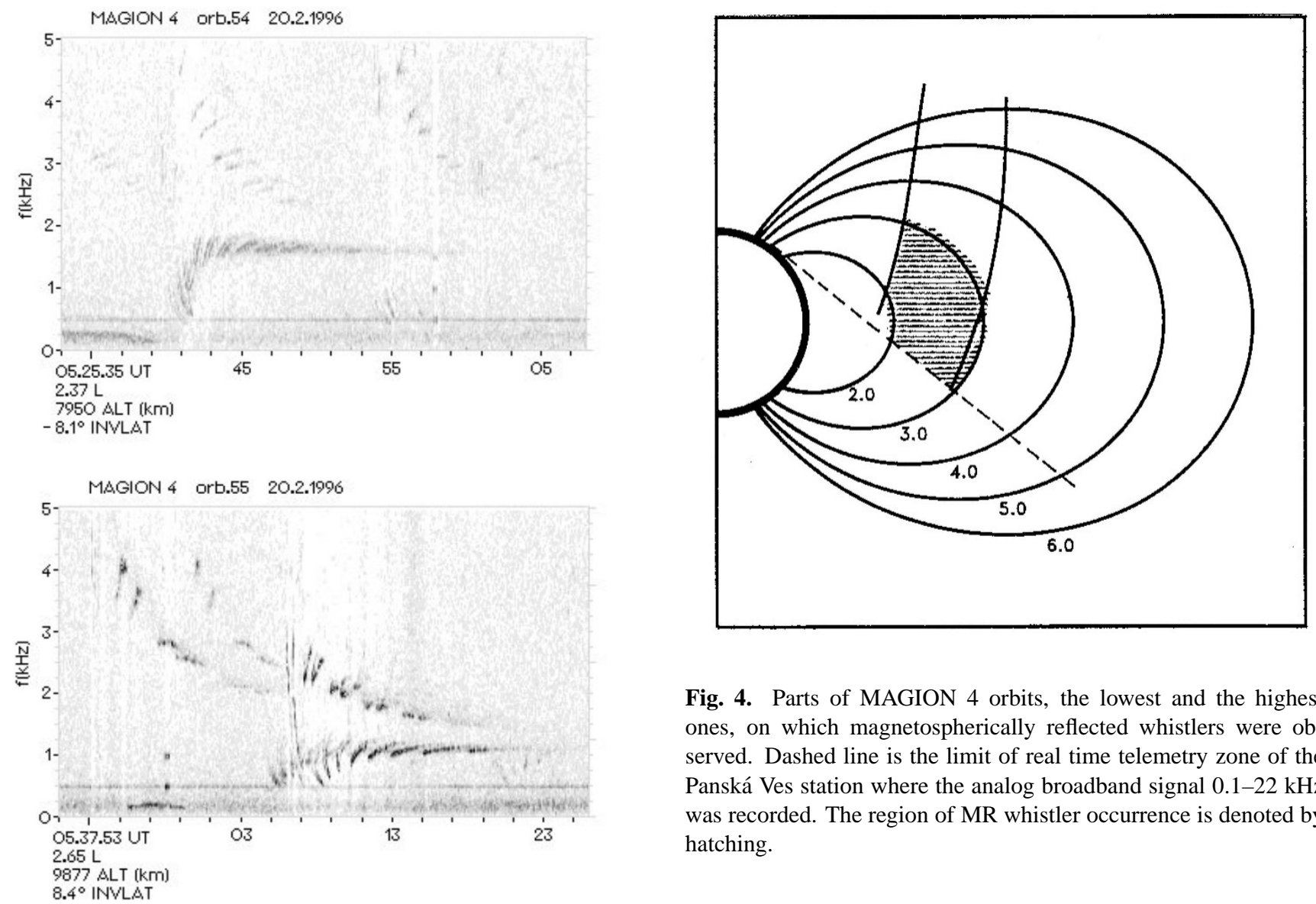

Fig. 4. Parts of MAGION 4 orbits, the lowest and the highest ones, on which magnetospherically reflected whistlers were observed. Dashed line is the limit of real time telemetry zone of the Panská Ves station where the analog broadband signal $0.1-22 \mathrm{kHz}$ was recorded. The region of MR whistler occurrence is denoted by hatching.

Fig. 3. Examples of more complicated types of MR whistlers recorded on the same orbit of MAGION 4, where the MR whistler shown in the upper left panel of Fig. 2 has been received.

ing a partial wave leakage from the duct and underdeveloped MR whistlers. Even when whistlers appear at the threshold of sensitivity, they are connected with the narrow LHR noise band.

Most of the data on LHR noise is related to observations on low-orbiting satellites. While it was always suggested that the lower frequency cutoff of LHR noise band was connected with the local LHR frequency in the vicinity of the satellite, a quantitative comparison was not easy due to the lack of ion concentration measurements. In the case of MAGION 5, the magnetospheric parts of the satellite path are at the heights above $5000 \mathrm{~km}$, where protons are the predominant ions. LHR noise has most often been observed by MAGION 5 from $L \simeq 2$ to $L \simeq 3.5$, i.e. in the range of $L$-shells where MR whistlers are usually found. This confirms the conclusion drawn by many researchers (e.g. Laaspere and Johnson, 1973) that nonducted energy of MR whistlers is the source of LHR noise. The experimental result of MAGION 5 on the predominant $L$ range of MR whistler occurrence can be understood if one takes into account that whistler mode waves enter the magnetosphere at lower $L$-shells first, then

go up to higher $L$-shells where they become magnetospherically trapped; at higher latitudes, not only the number of lightnings usually decreases, but also the waves entering the magnetosphere at large $\mathrm{L}$ have a strong tendency to bend towards lower $L$-shells (see Shklyar and Jiř́čcek, 2000).

\section{The features of nonducted whistler wave propagation in the plasmasphere}

In the framework of geometrical optics, the propagation of whistler mode waves is determined by the dispersion relation

$$
\begin{aligned}
\omega^{2}= & \omega_{\mathrm{LH}}^{2} \frac{k^{2}}{k^{2}+q^{2}}+\omega_{H}^{2} \frac{k_{\|}^{2} k^{2}}{\left(k^{2}+q^{2}\right)^{2}} \\
& \equiv \frac{\omega_{\mathrm{LH}}^{2}}{\left(1+q^{2} / k^{2}\right)}+\frac{\omega_{H}^{2} \cos ^{2} \theta}{\left(1+q^{2} / k^{2}\right)^{2}} .
\end{aligned}
$$

Here $\omega_{\mathrm{LH}}$ is the lower hybrid resonance frequency

$$
\omega_{\mathrm{LH}}^{2}=\frac{1}{M_{\mathrm{eff}}} \frac{\omega_{p}^{2} \omega_{H}^{2}}{\left(\omega_{p}^{2}+\omega_{H}^{2}\right)}, \quad \frac{1}{M_{\mathrm{eff}}}=\frac{m_{e}}{n_{e}} \sum_{\text {ions }} \frac{n_{\alpha}}{m_{\alpha}} .
$$

$n_{e}, m_{e}$ are electron concentration and mass, respectively; $n_{\alpha}, m_{\alpha}$ are the same for ions of species $\alpha ; k^{2}=k_{\|}^{2}+k_{\perp}^{2}$ where $k_{\|}$and $k_{\perp}$ are components of the wave normal vector parallel and perpendicular to the ambient magnetic field; 

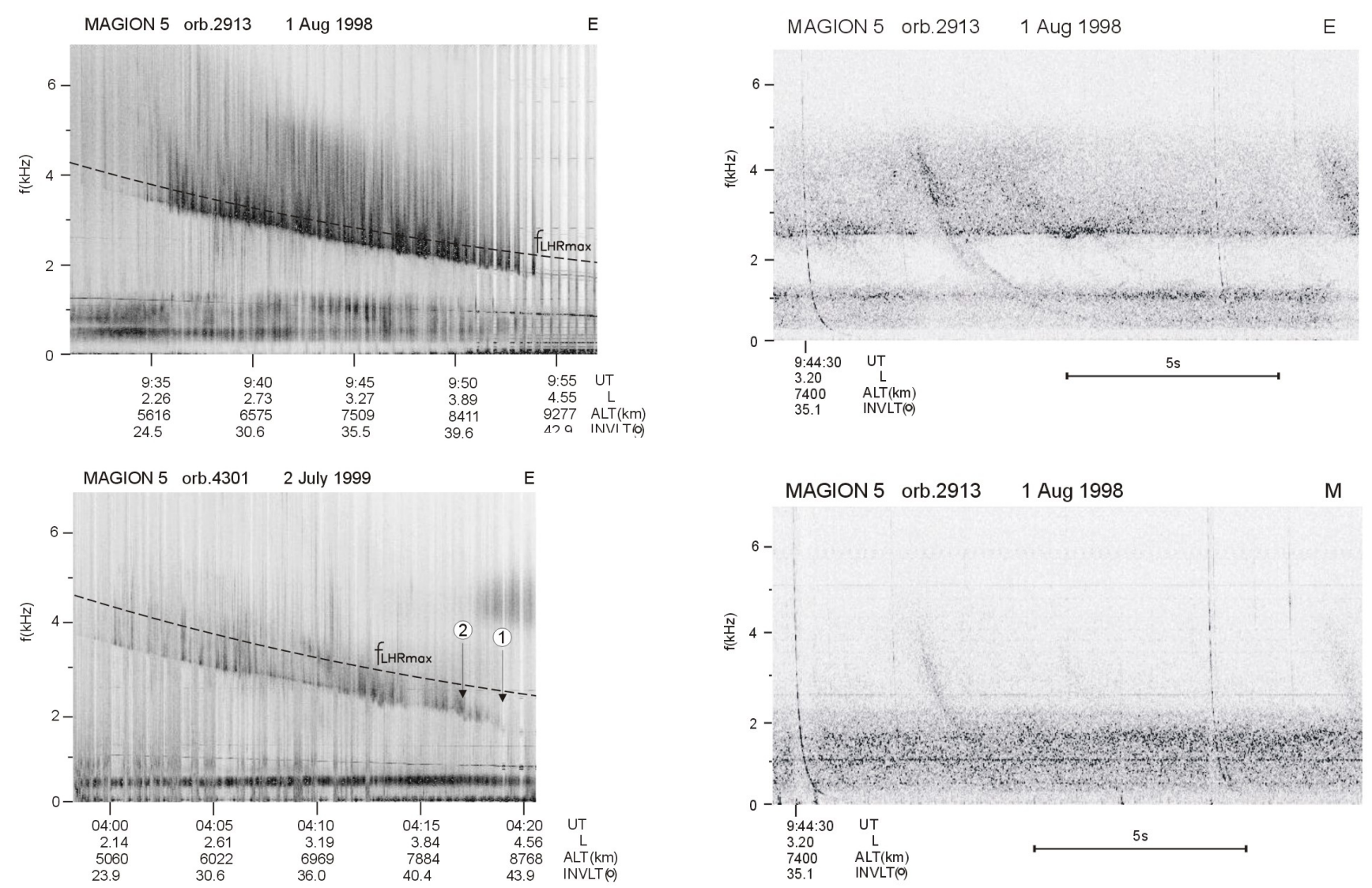

Fig. 5. Examples of LHR-noise recorded by the electric antenna in the plasmasphere.

Fig. 6. VLF wave spectrograms from a short interval of the MAGION 5 orbit No. 2913 (see Fig. 5, upper panel). Electric and magnetic components are shown in the upper and lower panels, respectively. LHR noise band is observed in the E- component only.

$q^{2}=\omega_{p}^{2} / c^{2} ; \omega_{p}$ and $\omega_{H}$ are electron plasma frequency and gyrofrequency, respectively; $c$ is the speed of light, and $\theta=\cos ^{-1}\left(k_{11} / k_{\perp}\right)$. Whistler mode waves described by the dispersion relation (1) have different character depending on the parameter $k^{2} / q^{2}$. For $k^{2} / q^{2} \lesssim 1$, which corresponds to quasi-longitudinal mode of propagation (Ratcliffe, 1959; Helliwell, 1965), the wave magnetic field dominates the wave electric field; at the same time, the wave group velocity is of the order of $\omega / k \sim c \omega_{H} \omega_{p}$. For $k^{2} / q^{2} \gg 1$, the wave becomes quasi-electrostatic and its group velocity essentially decreases. The corresponding regime of propagation is often referred to as a quasi-resonance one (e.g. Walker, 1976; Alekhin and Shklyar, 1980). In general, whistler mode waves exist at $\omega>\omega_{\mathrm{LH}}$, as well as $\omega<\omega_{\mathrm{LH}}$; however, the last range is accessible only for waves in quasilongitudinal mode of propagation. As for quasi-resonance waves having $k^{2} \gg q^{2}$, they do not propagate in the regions where $\omega$ is significantly smaller than $\omega_{\mathrm{LH}}$, while $\omega \simeq \omega_{\mathrm{LH}}$ is achieved only for the wave normal angles $\theta$ close to $\pi / 2$.

It is instructive to consider a typical whistler mode wave trajectory in the magnetosphere, and the variation of important wave parameters along the ray path. Figure 8 shows the ray trajectory for $5-\mathrm{kHz}$ wave launched vertically from a $500 \mathrm{~km}$ altitude at a latitude of $\lambda=-45^{\circ}$, and Fig. 9 provides

a summary of the propagation features for this wave. We see that initially the wave propagates in quasi-longitudinal regime and moves towards increasing $L$-shells. However, in the course of magnetospheric propagation, the wave normal angle $\theta$ becomes very close to $\pi / 2$; the parameter $k^{2} / q^{2}$ essentially increases, so that the wave becomes quasi-electrostatic and enters the quasi-resonance regime of propagation (Walter and Angerami, 1969; Kimura, 1985). As was mentioned above, a quasi-resonance wave does not propagate in the region where its frequency $\omega$ is essentially smaller than $\omega_{\mathrm{LH}}$, and is reflected at $\omega \lesssim \omega_{\mathrm{LH}}$. Thus, the wave becomes trapped in the region where $\omega>\omega_{\mathrm{LH}}$. At the same time, the transversal group velocity $v_{g \perp}$ becomes negative and very small in comparison with typical $v_{g \|}$ (which is violated only in the vicinity of the "reflection" point where $v_{g \|}$ $=0$ ). Thus, at this stage, the wave propagates predominantly along the field line and slowly moves towards lower $L$-shells $\left(v_{g \perp}<0\right.$. $)$ This general tendency of nonducted waves to enter the quasi-resonance regime of propagation and to finally move towards lower $L$-shells is an essential feature of whistler wave propagation in the plasmasperic region of magnetosphere, which helps to understand the spectrograms of non- 


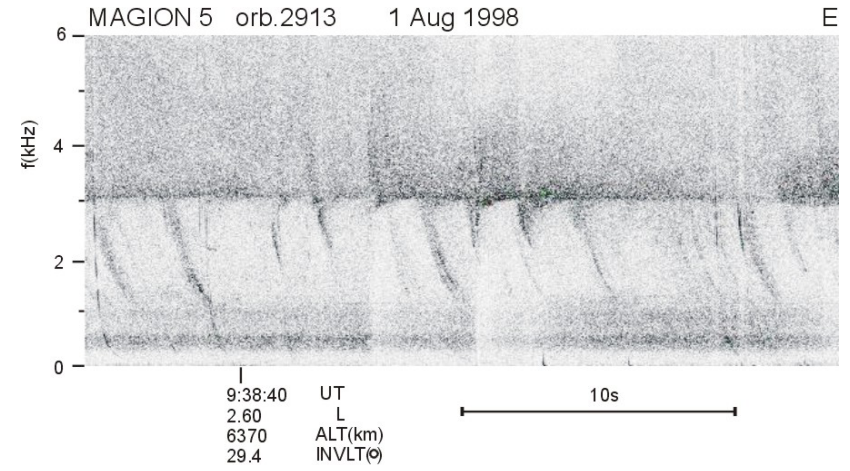

Fig. 7. 28 second interval from electric field registrations shown in the top panel of Fig. 5. A relationship between LHR noise and nonducted whistler mode waves is evident.

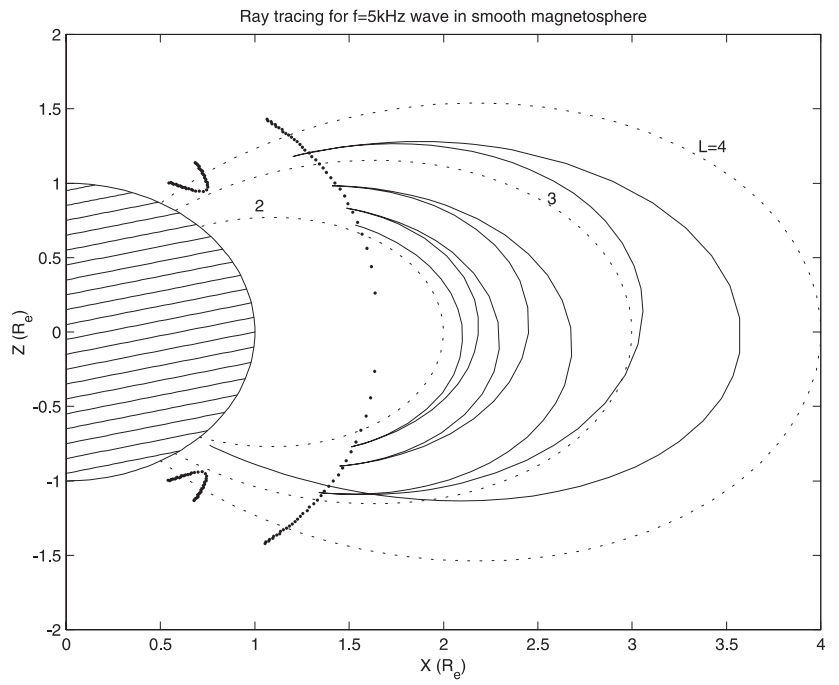

Fig. 8. Propagation paths for the wave with frequency $f=5 \mathrm{kHz}$ calculated without taking into account the plasmapause. The contour corresponding to LHR frequency $5 \mathrm{kHz}$ in the model used in calculations is shown by dotted line.

ducted waves like MR whistlers and Nu whistlers (Smith and Angerami, 1968).

\section{Some results on MR whistler modelling}

Observations onboard MAGION 4 and 5 have provided extensive original data on nonducted VLF waves and stimulated new studies of these phenomena, in particular, MR whistlers. In an attempt to gain an understanding of general properties of MR whistler spectrograms determined only by propagation features in a smooth magnetosphere, a computer program has been developed that accumulates the results of ray tracing calculations for a representative $2 \mathrm{D}$ continuum of wave trajectories, which permits one to generate, in one step, a spectrogram for a given observation point and illuminating region (Shklyar and Jiř́čcek, 2000). In the ray tracing, the
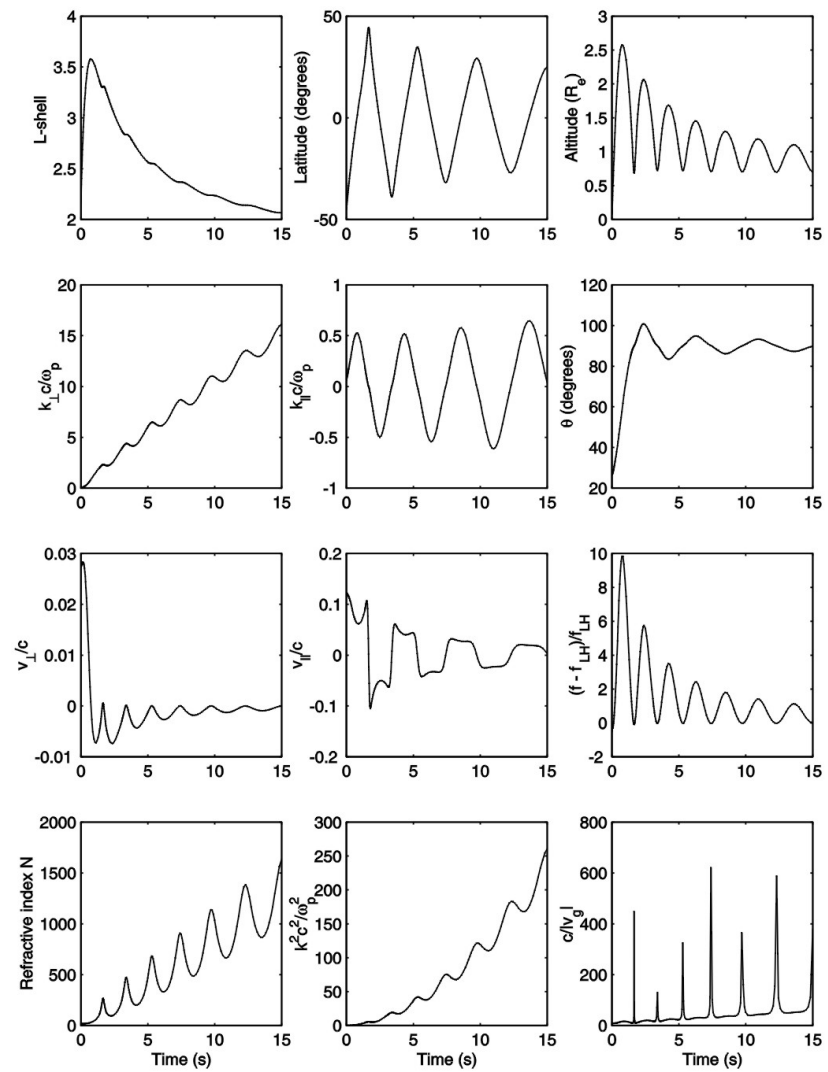

Fig. 9. Essential parameters characterizing the wave propagation shown in Fig. 8.

dipolar model of the ambient magnetic field and gyrotropic $\left(\omega_{p}^{2} \propto \omega_{H}\right)$ model of plasma density have been used. The undertaken computer simulations, together with the analytical consideration, permitted one to reproduce and explain a number of features of MR whistler spectrograms. Those features are:

1. Spectrograms observed at higher $L$-shells $(L \gtrsim 3)$ are poor in high frequencies. The higher frequencies correspond to the waves originating at larger $L$-shells, while the lower frequencies are related to lower initial $L$-shells of the illuminating region (Edgar, 1976).

2. All clearly defined traces on the spectrograms are formed by the wave packets making the same number of hops before reaching the satellite.

3. The nose frequency on the spectrogram corresponding to a minimal time on a given trace is formed by the wave with $v_{g \perp}=0$ at finite wave normal angle.

A detailed account of all these (as well as some other) features may be found in the paper by Shklyar and Jiříček (2000). Qualitatively, they can be understood on the basis of the wave propagation properties discussed in Sect. 3. The first two features are connected with the quality of quasiresonance waves that finally move towards lower $L$-shells, 

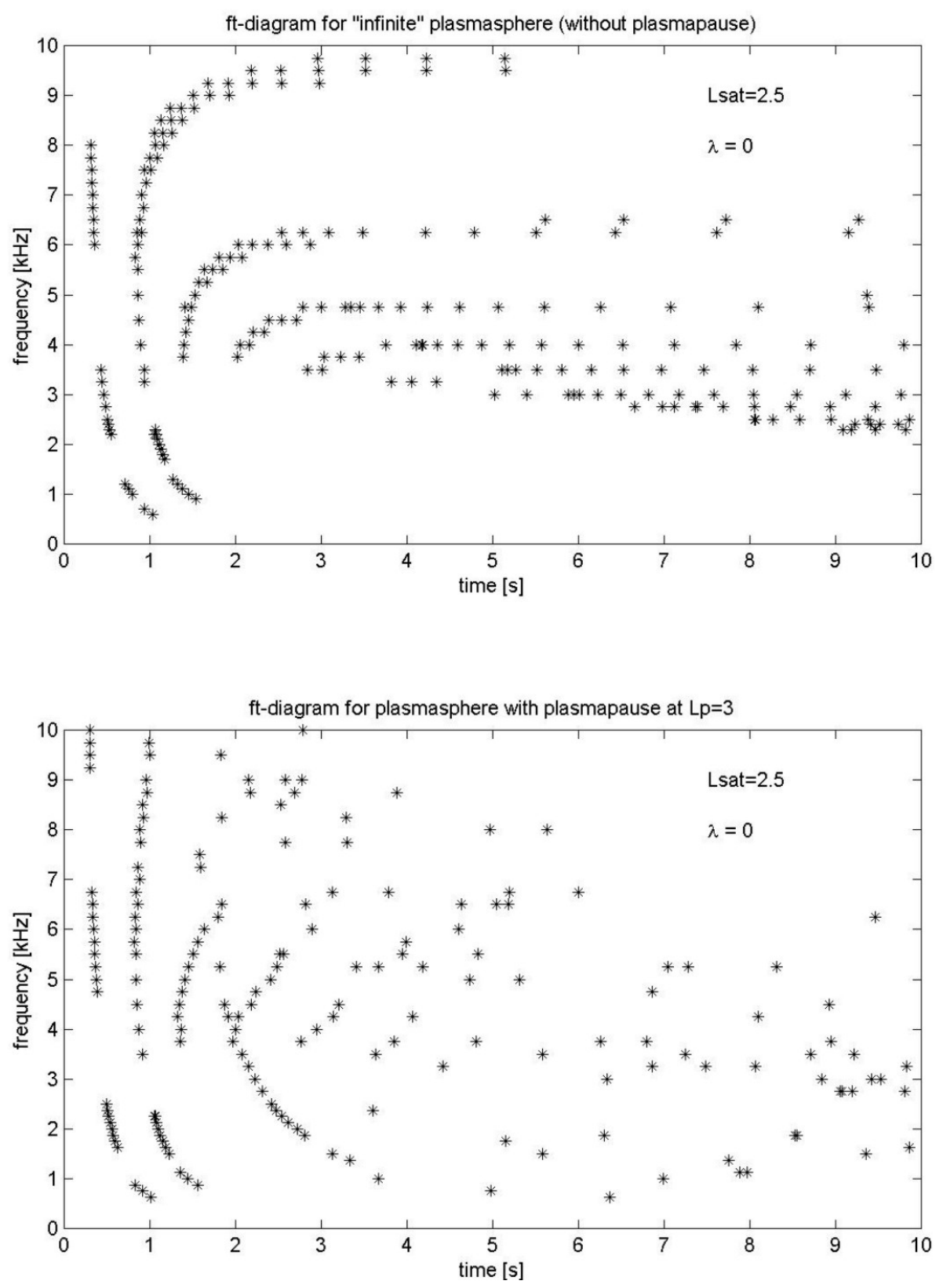

Fig. 10. Comparison of simulated MR whistler spectrograms with (lower panel) and without (upper panel) taking into account the plasmapause, in the case when a satellite is below the plasmapause. and this tendency is more pronounced for higher frequency waves. That is why, if a spectrogram is taken at high $L$ shells, most of the high frequency waves appear to propagate in bounce-oscillating regime below the satellite, while those which are observed should have come from higher latitudes. Feature number 2 is, after all, connected with a continuous dependence of the time of propagation to the observation point on the initial latitude and wave frequency. Feature number 3 is the manifestation of the dispersion property of whistler mode waves, which consists in the existence of maximum longitudinal group velocity corresponding to $k^{2} / q^{2} \sim 1, v_{g \perp}=0$ and $\theta \neq 0$ (the analog of Gendrin angle (Gendrin, 1961) for the dispersion relation with the account of finite LHR frequency). In the present study, we raise a new, important aspect of the problem of MR whistlers. As follows from the experimental results, MR whistlers are hardly observed in magnetospherically disturbed conditions, when the plasmapause is at lower $L$-shells. This is in line with the understanding gained from both analytical and numerical analysis that an extended smooth plasmasphere is necessary to obtain a full pattern of MR whistler spectrogram. In the simulations by Shklyar and Jiříček (2000), however, the plasmapause has not been taken into account. Here we extend these simulations by considering the consequences of the plasmapause.

For this aim, we have performed the calculations similar to that by Shklyar and Jiříček (2000), but with the following model of plasma density that takes into account the plasma- 

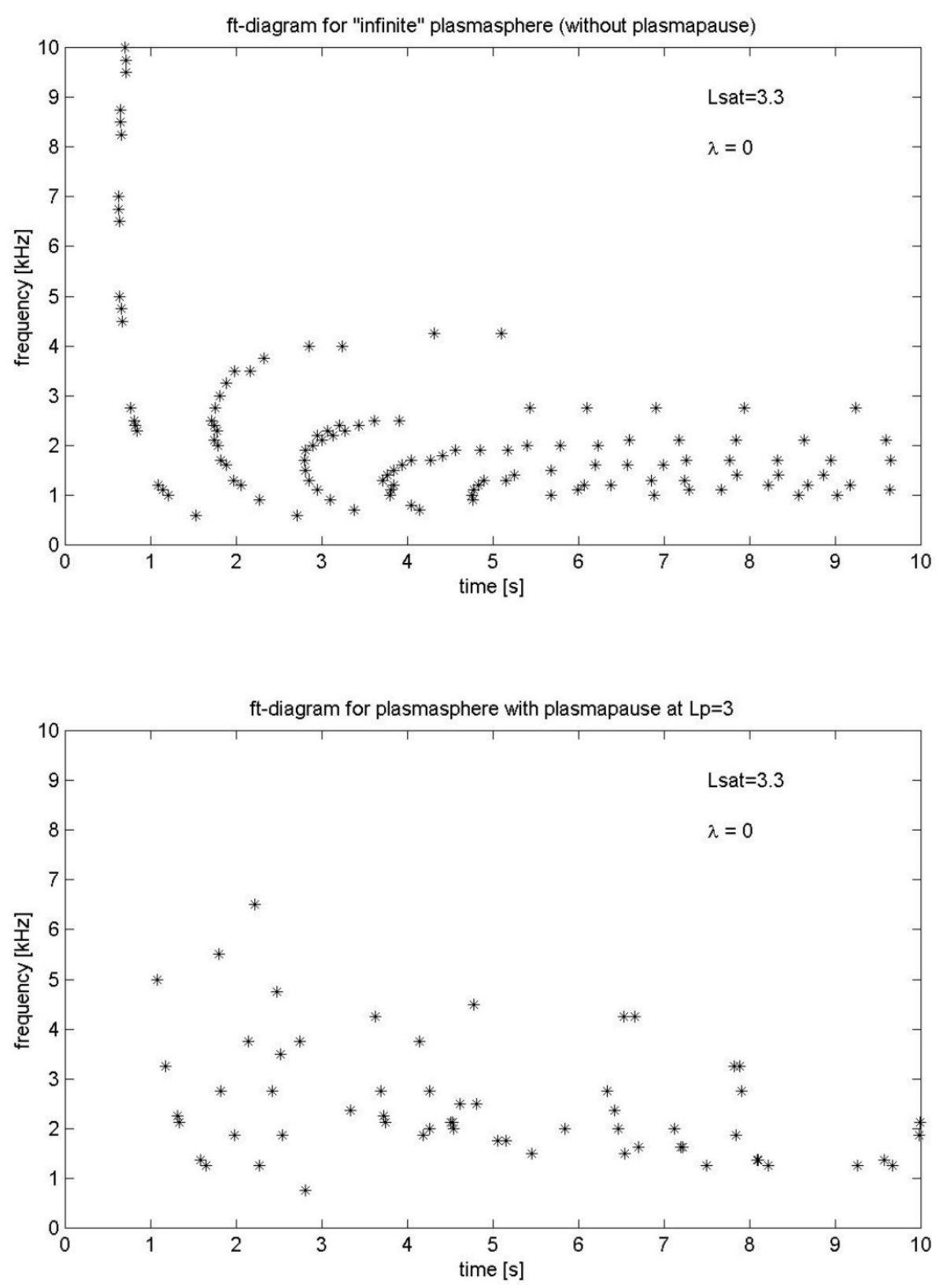

Fig. 11. Comparison of simulated MR whistler spectrograms with (lower panel) and without (upper panel) taking into account the plasmapause, in the case when a satellite is above the plasmapause. pause:

$\omega_{p}^{2} \propto \omega_{H}^{n} \cdot P(L)$

The factor $P(L)$ which enters the expression (3) for $\omega_{p}^{2}$ (thus, for plasma density) and models the plasmapause is chosen in the form:

$P(L)=(1-p) \exp \left(-e^{\frac{L-L p}{\delta L}}\right)+p$.

The quantity $P(L)$ depends only on $L$ and is determined by three parameters: $L_{p}, \delta L_{p}$ and $p$, which have clear physical meaning, namely, $p$ is the value of $P(L)$ beyond the plasmapause; $L_{p}$ is the position of the plasmapause where $P(L)$ takes the value

$p<p+(1-p) e^{-1} \equiv 1-(1-p)\left(1-e^{-1}\right)<1$ (provided that $p<1$, of course), and $\delta L_{p}$ is the characteristic width of the plasmapause. These three parameters make it possible to model the plasmapause quite well in most "onedrop" cases.

It is easy to see that $P(L)$ is close to 1 for $L<L_{p}-\delta L_{p}$ and takes another (smaller) constant value at $L>L_{p}+\delta L_{p}$. Thus, before and beyond the plasmapause, the cold plasma density in this model is proportional to the $n$-th power of gyrofrequency.

The influence of the plasmapause as infered from mumerical modelling is illustrated by Figs. 10 and 11. In these simulations, the following values of parameters have been used: $n=1 ; L_{p}=3 ; \delta L_{p}=0.2 ; p=0.15$. In Fig. 10, the satellite position is at $L=2.5$ at the equator, and the 


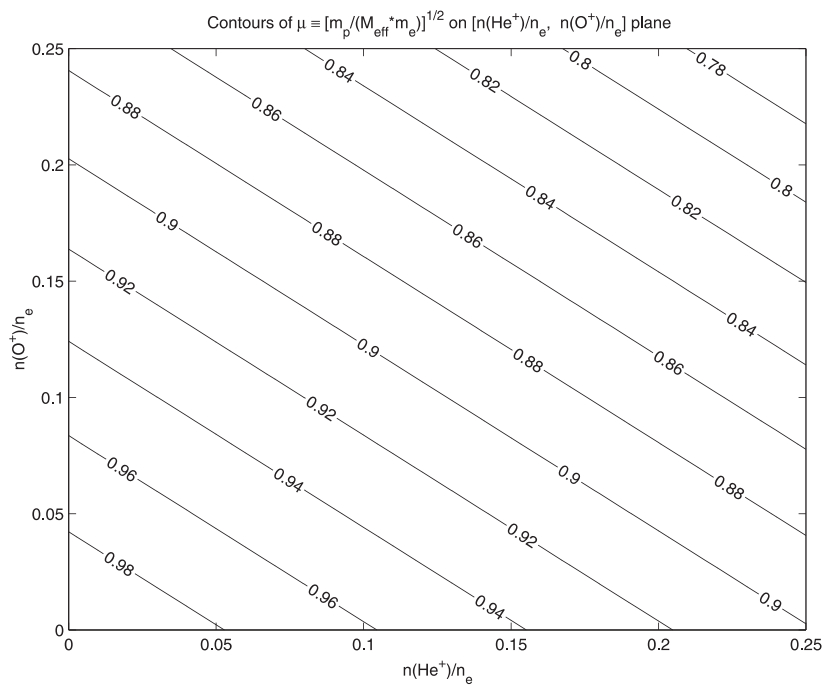

Fig. 12. LHR frequency contours in a densed three component plasma.

illuminating region is assumed to be spread in the latitude range $25^{\circ}-50^{\circ}$ at the height of $500 \mathrm{~km}$. The spectrogram in the upper panel of Fig. 10 is calculated without taking into account the influence of the plasmapause, and in the lower panel, the plasmapause at $L_{p}=3$ is introduced into consideration as described above. We see that the large number of traces of MR whistler are completely destroyed in the presence of plasmapause, since sharp gradients remove a smooth dependence of propagation characteristics on initial conditions. The organization and the parameters used in the modelling, shown in Fig. 11, are the same as in Figure 10, but a satellite is located at $L=3.3$. Thus, in the case shown in the lower panel, the satellite is above the plasmapause, which is still at $L=3$. One can see an essential degradation of the spectrogram when a plasmapause is present, especially when the satellite is above the plasmapause.

\section{What can be infered from geometrical optics concerning LHR noise}

Although the problem of LHR noise cannot be completely reduced to geometrical optics, and although linear and nonlinear wave conversion, as well as wave generation processes should play a part, it is first of all necessary to explore the consequences of the consideration in the frame of geometrical optics. (In this paper, we do not consider at all the processes mentioned above.) We suppose that LHR noise represents a kind of wave which is always present in the magnetosphere. Obviously, the first candidates are the whistler mode waves, excited by lightnings, which are then trapped, due to the magnetospheric reflections, and thus, fill up the magnetosphere.

As for other sources of LHR noise, in particular, concerning wide band auroral and plasmaspheric hiss, we should say

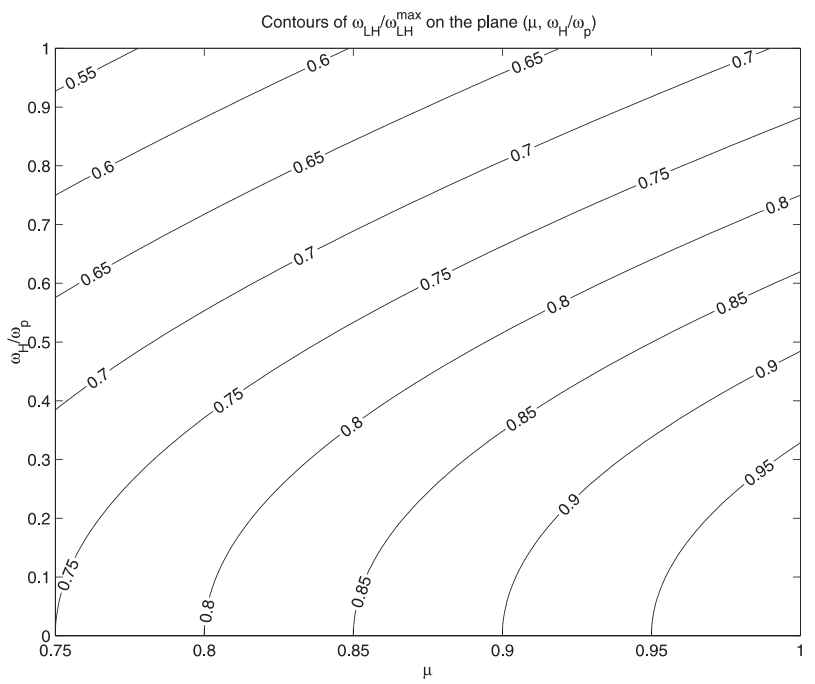

Fig. 13. LHR frequency for various ion composition and finite values of $\omega_{H} / \omega_{p}$.

the following. According to our observations, the frequency band of plasmaspheric hiss is in the range below $1.5 \mathrm{kHz}$, which is lower than LHR frequency on MAGION 4 and 5 paths where LHR noise is observed. As far as the wide band auroral hiss is concerned, we do not rule out a possible relation between this emission and LHR noise. However, unfortunately, due to technical reasons, the observations of MAGION 4 and 5 are confined to $L<7$, where auroral hiss is not observed.

Returning to consideration of the whistler mode waves, we remember that they may propagate at frequencies $\omega>\omega_{\mathrm{LH}}$, as well as at $\omega<\omega_{\text {LH }}$. Why then does the spectrum usually observed in the experiment have a pronounced maximum at $\omega=\omega_{\mathrm{LH}}$ ? To explain this, we briefly recall the arguments which have been used in many considerations. While nonducted whistler mode waves propagate in smooth plasmasphere, they naturally turn into quasi-resonance mode, where their group velocity essentially decreases. From the energy conservation, which requires the energy flux to be constant along the ray path, one can easily understand that the region of minimum group velocity should correspond to maximum wave amplitude. It appears that the minimum group velocity of the wave is achieved in the region where the wave frequency is very close to the local LHR frequency. In reality, since $v_{g \perp}$ is very small, the minimum $\left|v_{g}\right|$ is achieved in a close vicinity of the point where $v_{g \|}=0$. As follows from (1), it takes place at

$\omega=\omega_{H}\left(\frac{k^{2}}{k^{2}+q^{2}}\right)^{1 / 2}$

which, for typical values of the parameter $k^{2} / q^{2}$ at the reflection point, is very close to $\omega_{\mathrm{LH}}$ (see Fig. 9). However, the researchers often found that the spectrum lies below local LHR frequency. Since the finite values of $k^{2} / q^{2}$ can hardly 
account for this, a possible reason may be that the lower frequency cutoff is often compared with the LHR frequency calculated in the assumptions of purely proton, dense $\left(\omega_{p}^{2} \gg\right.$ $\omega_{H}^{2}$ ) plasma, which provides the maximum possible value of $\omega_{\mathrm{LH}}$ in a given magnetic field, equal to $\left(m_{e} / m_{p}\right)^{1 / 2} \omega_{H}$, which we denote as $\omega_{\mathrm{LH}}^{\max }$. If any, or both, of the assumptions mentioned above is violated, the real LHR frequency decreases. For example, in the case of one component plasma, if the quantity $\omega_{p}^{2} / \omega_{H}^{2}$ drops from 10 to 5 (or 3), then $\omega_{\mathrm{LH}}$ changes from $0.95 \omega_{\mathrm{LH}}^{\max }$ to $0.91 \omega_{\mathrm{LH}}^{\max }$ (or $0.87 \omega_{\mathrm{LH}}^{\max }$ ). The presence of different ion species, other than protons, also leads to a decrease of $\omega_{\mathrm{LH}}$, as compared to $\omega_{\mathrm{LH}}^{\max }$. The influence of the ion effective mass on the LHR frequency in the case of three ion species is illustrated in Fig. 12, where the contours of the parameter

$\mu=\sqrt{\frac{m_{p}}{m_{e}} M_{\mathrm{eff}}} \equiv \frac{\omega_{\mathrm{LH}}}{\omega_{\mathrm{LH}}^{\max }}\left(1+\frac{\omega_{H}^{2}}{\omega_{p}^{2}}\right)$

(see Eq. (2)) are plotted on the plane where $x$ - and $z$ axes represent the percentage of the helium and oxygen ions, respectively. A combined influence of both factors can be estimated with the help of Figs. 12 and 13. Figure 13 shows the contours of $\omega_{\mathrm{LH}}$ normalized to $\omega_{\mathrm{LH}}^{\max }$ in the $\left(\mu, \omega_{H} / \omega_{p}\right)$ plane. To use these graphs, one should first find the value of the parameter $\mu$ with the help of the plot in Fig. 12, and then find the value of $\omega_{\mathrm{LH}} / \omega_{\mathrm{LH}}^{\max }$ using the plot in Fig. 13.

\section{Discussion and conclusions}

New observations of nonducted VLF waves onboard MAGION 4 and 5 stimulated interest to further studies of various phenomena connected with nonducted whistler propagation in which LHR effects are of principal importance. Two phenomena of this type which have been discussed in this study are MR whistlers and LHR noise.

In the investigation of MR whistlers, we proceed from general features of nonducted wave propagation in the plasmasphere, and turn from the analysis of ray trajectories into simulations of the whole spectrograms. Such spectrograms keep no traces of spectral intensity, but in return, they contain the information on the region of origin, the number of hops, and the direction of group velocity of the wave packets which form the spectrogram. The simulations performed together with the analytical consideration yield understanding of several features of MR whistler spectrograms, such as principal dependence of a spectrogram on the observation point rather than on the illuminating region; the correspondence of the nose frequency to the wave packet propagating along the geomagnetic field $\left(v_{g \perp}=0\right)$; the decrease of the nose frequency with increasing trace number, and others. The understanding of spectrogram formation and simplicity of calculating spectrograms, based on the developed program, permit to simulate, and thus, to explain rather complicated MR whistler spectrograms observed in the experiment. An important new point in the present study is the influence of the plasmapause, which leads to qualitative new features of the spectrogram and permits one to account for experimental observation that a full pattern of MR whistler spectrogram is found only in quiet magnetospheric conditions.

Recent registrations of LHR noise onboard MAGION 4 and 5 have confirmed the features of this noise band known from early satellite experiments: quasielectrostatic character of this emission, and lower cutoff at the local LHR frequency (see the spectrograms above). The region where LHR noise is detected usually coincides with that of MR whistlers. In the events where LHR noise is connected with "ducted" whistler traces, the noise band is registered only when the whistler trace has a diffusive character. Both of these results provide strong evidences that LHR noise is nothing but nonducted whistlers, or MR whistlers observed at their reflection point where the wave amplitude has a pronounced maximum.

The occurrence of $f_{\text {LHR }}$ values lower than the $f_{\text {LHRmax }}$ can be explained especially by the higher content of $\mathrm{He}^{+}$ ions. This is in agreement with DE-1 result which shows that there is a region in the plasmasphere above $\sim 4000 \mathrm{~km}$ and $L \simeq 2.0$ where the $\mathrm{He}^{+} / \mathrm{H}^{+}$density ratio has been observed to maintain a nearly constant value of approximately 0.2. (Newberry et al., 1989).

Acknowledgements. This study was supported by grant No A3042801 of the Grant Agency of the Academy of Sciences of the Czech Republic, by grant No 102/98/1141 of the Grant Agency of the Czech Republic, and by grant No 00-05-64503 of the Russian Foundation for Basic Research. D. R. Shklyar wishes to thank Upper Atmosphere Department of the Institute of Atmospheric Physics in Prague for hospitality and financial support during this study.

Topical editor G. Chanteur thanks M. Parrot and another referee for their help in evaluating this paper.

\section{References}

Alekhin, Ju. K. and Shklyar, D. R., Some questions of electromagnetic wave propagation in magnetosphere, Geomagnetism i Aeronomia, 20, 501-507, 1980.

Barrington, R.E. and Belrose, J. S., Preliminary results from the very-low-frequency receiver on board Canada's Alouette satellite, Nature, 188, 651-656, 1963.

Barrington, R. E., Belrose, J. S., and Kelley, D. A., Very-lowfrequency noise bands observed by the Alouette 1 satellite, J. Geophys. Res., 68, 6539, 1963.

Bell, T. F. and Ngo, H. D., Electrostatic waves stimulated by coherent VLF signals propagating in and near the inner radiation belt, J. Geophys. Res., 93, 2599, 1988.

Bell, T. F., Inan, U. S., Sonwalkar, V. S., and Helliwell, R. A., DE-1 observation of lower hybrid waves excited by VLF whistler mode waves, Geophys. Res. Lett., 18, 393, 1991.

Brice, N. M. and Smith, R. L., Recordings from satellite Alouette-2 - A very low frequency plasma resonance, Nature, 203, 926-927, 1964.

Brice, N. M. and Smith, R. L., Lower hybrid resonance emissions, J. Geophys. Res., 70, 71-80, 1965.

Burtis, W. J., Electron concentrations calculated from the lower hybrid resonance noise band observed by OGO 3, J. Geophys. Res., 78, 5515-5523, 1973. 
Carpenter, D. L. and Anderson, R. R., An ISEE/whistler model of equatorial electron density in the magnetosphere, J. Geophys. Res., 97, 1097-1108, 1992.

Edgar, B. C., The upper- and lower-frequency cutoffs of magnetospherically reflected whistlers, J. Geophys. Res., 81, 205-211, 1976.

Gendrin, R., Le guidage des whistlers par le champ megnetique, Planet. Space Sci., 5, 274-282, 1961.

Helliwell, R. A., Whistlers and related ionospheric phenomena, Stanford University Press, Stanford, Calif., USA, 1965.

Jiřriček, F. and Tříska, P., LHR-associated phenomena observed with the Interkosmos-10 satellite, Space Res., XVI, Akad.-Vlg, Berlin, 567, 1976.

Jiříček, F. and Shklyar, D. R., On the problem of quasi-resonance wave trapping in LHR waveguide, Internat. J. of Geomagn. Aeronomy, 1, 193-201, 1999.

Kimura, I., Effects of ions on whistler-mode ray tracing. Radio Sci. 1, 269-283, 1966.

Kimura, I., Whistler mode propagation in the Earth and planetary magnetospheres and ray tracing techniques, Space Sci. Rev., 42, 449-466, 1985.

Laaspere, T., Johnson, W. C., and Sempreron, L. C., Observations of auroral hiss, LHR noise, and other phenomena in the frequency range $20 \mathrm{~Hz}-540 \mathrm{kHz}$ on OGO 6, J. Geophys. Res., 76, 44774493, 1971.

Laaspere, T. and Johnson, W. C., Additional results from an OGO-6 experiment concerning ionospheric electric and electromagnetic fields in the range $20 \mathrm{~Hz}$ to $540 \mathrm{kHz}$, J. Geophys. Res., 78, 29262944, 1973.
Newberry, I. T., Comfort, R. H. , Richards, P. G., and Chappell, C. R., Thermal $\mathrm{He}^{+}$in the plasmasphere: comparison of observations with numerical calculations, J. Gephys. Res., 94, 15265 $15276,1989$.

Ratcliffe, J. A., The magneto-ionic theory and its applications to the ionospher, Cambridge Univ. Press, 1959.

Sazhin, S.S., Natural radio emissions in the Earth's magnetosphere, Nauka, Moscow, 1982, (in Russian).

Shklyar, D. R. and Jiříček, F., Simulation of nonducted whistler spectrograms observed aboard the MAGION 4 and 5 satellites, Journal of Atmospheric and Solar-Terrestrial Physics, 62, 347370, 2000.

Smith, R. L., and Angerami, J.J., Magnetospheric properties deduced from OGO 1 observations of ducted and nonducted whistlers, J. Geophys. Res., 73, 1-20, 1968.

Thorne, R. M. and Horne, R. B., Landau damping of magnetospherically retlected whistlers, J. Geophys. Res., 99, 17 249-17 258, 1994.

Titova, E. E., Di, V. I., Yurov, V. E., Raspopov, O. M., Trakhtengertz, V. Yu., Jiříček, F., and Tříska, P., Interaction between VLF waves and turbulent ionosphere, Geophys. Res. Lett., 11, 323, 1984.

Walker, A. D. M., The theory of whistler propagation, Revs. Geophysics, 14, 629-638, 1976.

Walter, F. and Angerami, J. J., Nonducted mode of VLF propagation between conjugate hemispheres; observations on OGO's 2 and 4 of the "walking-trace" whistler and of Doppler shifts in fixed frequency transmissions, J. Geophys. Res., Space Phys., 74, 6352-6370, 1969. 